\title{
H-Mode Threshold and Confinement in Helium and Deuterium in ASDEX Upgrade
}

\author{
F. Ryter, T. Pütterich, M. Reich, A. Scarabosio, E. Wolfrum, \\ R. Fischer, M. Gemisic Adamov, N. Hicks, B. Kurzan, C. Maggi, \\ R. Neu, V. Rohde, G. Tardini and ASDEX Upgrade TEAM \\ Max-Planck-Institut für Plasmaphysik, EURATOM Association, D-85748 Garching \\ E-mail: ryter@ipp.mpg.de
}

\begin{abstract}
In 2008, experiments have been carried out in ASDEX Upgrade to compare Hmode power threshold and confinement time in helium and deuterium. A scan in magnetic field and a wide density variation indicate that the threshold power in the two gases is very similar. The density dependence of the threshold exhibits a clear minimum. Confinement in helium is about $30 \%$ lower than in deuterium, mainly due to the reduction of the ion density caused by $\mathrm{Z}=2$ in helium.
\end{abstract}

PACS numbers: 52.55.Fa, 52.50.Gj, 52.50.Sw

\section{Introduction}

The H-Mode is at present the baseline scenario foreseen for ITER and its threshold power $\left(P_{t h r}\right)$ predicted by the scaling deduced from the ITPA database is about $50 \mathrm{MW}$ in deuterium at a density of $5 \times 10^{19} \mathrm{~m}^{-3}$ [1]. It has been emphasized recently that achieving the $\mathrm{H}$-mode during the non-nuclear phase of ITER in hydrogen or helium plasmas is highly desirable to assess several physical and technical issues. However, it is well established that $P_{t h r}$ in hydrogen is about 2 times larger than in deuterium, [2, 3, 4, 5]. In contrast, it has been shown in JET that $P_{t h r}$ in helium is only about $40 \%$ higher than in D, [6]. This motivated in April 2008 the ITPA Transport and Confinement Topical Group to initiate Joint Experiments (proposal TC-4 H-Mode transition and confinement dependence on ionic species), including ASDEX Upgrade, DIII-D and JET, to better assess H-mode threshold and confinement dependencies on ion species. To this aim, dedicated experiments in ASDEX Upgrade have been performed between May and October 2008. Moreover, the existence of a minimum threshold $\left(P_{t h r, \min }\right)$ at a density $n_{\min }$ was observed in several tokamaks $[7,8,9,1]$. Results from Alcator C-Mod suggesting that $n_{\text {min }}$ increases with magnetic field, [10], are a concern for ITER at $B_{T} \approx 5$ $\mathrm{T}$. This issue is addressed in the ITPA proposal TC-3 (Scaling of the low density limit of the H-Mode threshold), for which experiments in ASDEX Upgrade have also been carried out in 2008. In this Letter we present the ASDEX Upgrade contributions to both TC-3 and TC-4. 


\section{Previous results in $\mathrm{H}, \mathrm{D}$ and ${ }^{4} \mathrm{He}$}

Besides the well-documented deuterium results on threshold and confinement contributed to the ITPA data bases for about 15 years, a rather complete hydrogen data set was available from ASDEX Upgrade before 2008, but only sparse results from non-systematic experiments in ${ }^{4} \mathrm{He}$ existed. In Fig. 1, the threshold results for the three gases are compared using the ITPA scaling expression [1], only valid above $n_{\min }$ and which reads for $\mathrm{D}$, in MW:

$$
P_{\text {thr,scal } 08}=0.049 B_{T}^{0.80} n_{20}^{0.72} S^{0.94}
$$

where $B_{T}[T], n_{20}\left[10^{20} \mathrm{~m}^{-3}\right]$ and $S\left[\mathrm{~m}^{2}\right]$ are respectively the magnetic field , line-averaged density and plasma surface area. The values for $P_{t h r}$ are provided by the usual net heating power just prior to the LH transition and defined as

$$
P_{\text {net }}=P_{\text {Ohm }}+P_{\text {heat }}-d W / d t
$$

where $P_{O h m}$ is the residual Ohmic power, $P_{\text {heat }}$ the absorbed auxiliary heating power and $W$ is the plasma energy content. As usually done for such studies and for the scaling as well, the radiation losses are not subtracted. It should be underlined that the radiated power from the main plasma corresponds to about $30 \%$ of the input power in the discharges studied here and is very similar in deuterium and helium.

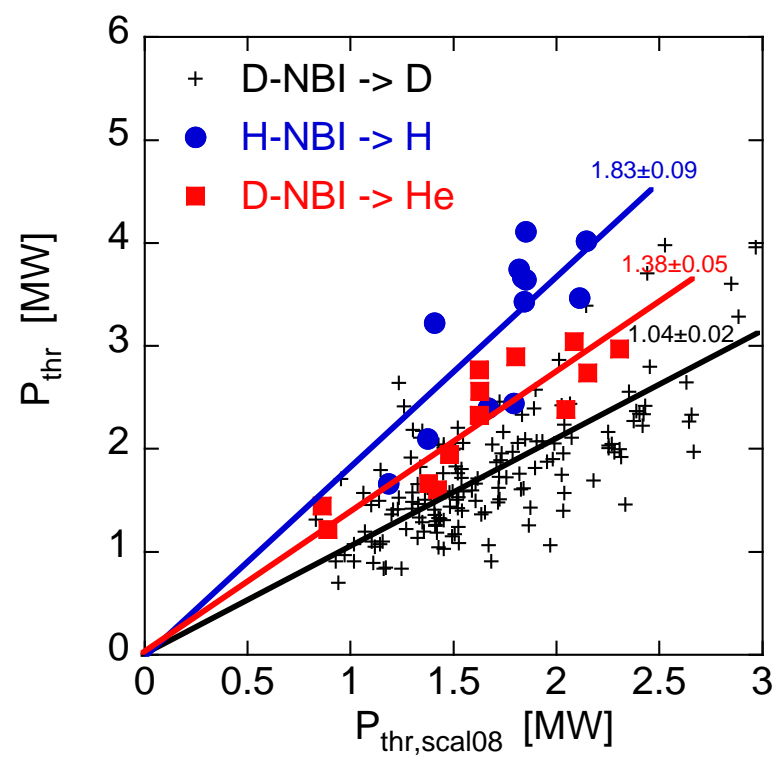

Figure 1. Power threshold results in ASDEX Upgrade in D, H and He before 2008. Experimental power threshold versus scaling assuming deuterium. The lines are linear fits forced through the origin.

Figure 1 indicates that the deuterium data are in good agreement with the scaling as shown by the slope of the fit which is very close to 1 . The ranges in our dataset are $1.5-2.8$ $\mathrm{T}$ for $B_{T}, 0.5-1.0 \mathrm{~m}^{-3}$ for $n_{20}$ and $42-45 \mathrm{~m}^{2}$ for $S$. The threshold for $\mathrm{H}$ is about 1.8 higher than in $\mathrm{D}$, whereas that of ${ }^{4} \mathrm{He}$ plasmas only by about 1.4. The $\mathrm{D}$ and $\mathrm{H}$ confinement data (not 
shown here) agree with the usual confinement scaling ITERH98y2, [11], including the mass dependence $M^{0.19}$. The ${ }^{4} \mathrm{He}$ confinement data are about $30 \%$ lower than in D and very close to those in $\mathrm{H}$. These previous threshold and confinement results are in agreement with those from JET, [6].

\section{New threshold and confinement results in ${ }^{4} \mathrm{He}$ and D}

\subsection{Experimental conditions}

The new helium studies have been carried out in July 2008. Electron Cyclotron Heating and Hydrogen Neutral Beam Injection were used to heat the plasmas. Since 2008, the ECH system delivers up to $2.2 \mathrm{MW}$ absorbed in the plasma, which allows to explore H-mode threshold over a wide density window. In particular, the low density branch of $P_{t h r}$ could be extensively investigated. The choice of hydrogen for NBI has been governed by the fact that this species will be used in ITER at the beginning and that ASDEX Upgrade ${ }^{4} \mathrm{He}$ data with D-NBI already existed. As adding hydrogen into helium plasmas is expected to increase $P_{t h r}$ and reduce confinement, this is a conservative approach for extrapolating to ITER in which the NBI fueling will be much lower than in ASDEX Upgrade. Figure 2 shows the helium purity, measured by spectroscopy in the divertor region and assessed by active charge exchange spectroscopy in the main plasma for some of the points.

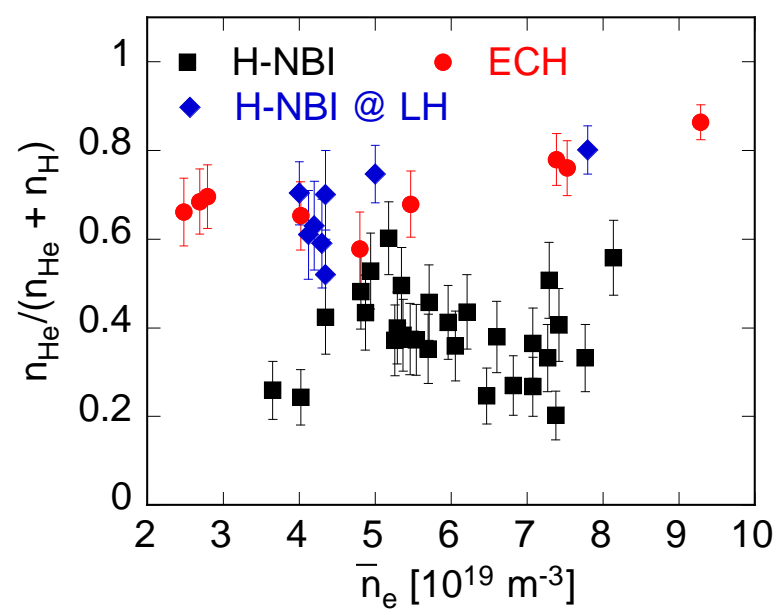

Figure 2. Purity of ${ }^{4} \mathrm{He}$ versus density, for ECH any time during discharge, for H-NBI at LH transition or any time in discharge.

The helium purity was around $75 \%$ for ECH-heated plasmas with a trend to increase with density, attributed to gas puffing. For plasmas heated with H-NBI, the helium purity is lower and varies strongly. It should be noted that in general with $\mathrm{H}-\mathrm{NBI}$, the ${ }^{4} \mathrm{He}$ concentration decreases with time during a discharge, while in counterpart the $\mathrm{H}$ concentration increases. This is the cause for the large scatter of the H-NBI data taken at any time during the discharges, with different values of heating power (black squares in Fig. 2). In contrast, the H-NBI points 
at the LH transition have a rather high helium purity because they are early in the discharges and with rather low NBI power.

\subsection{Power threshold in $\mathrm{D}$ and ${ }^{4} \mathrm{He}$}

The $B_{T}$ dependence of $P_{t h r}$ in ${ }^{4} \mathrm{He}$ has been studied using H-NBI at $\bar{n}_{e} \approx 4 \times 10^{19} \mathrm{~m}^{-3}$. As shown in Fig. 3, the $B_{T}$ dependence of $P_{t h r}$ in ${ }^{4} \mathrm{He}$ follows that of the scaling. On average, the 2008 points are somewhat below the scaling, as discussed later. For comparison, we also plotted data points from previous deuterium plasmas in the same density range.

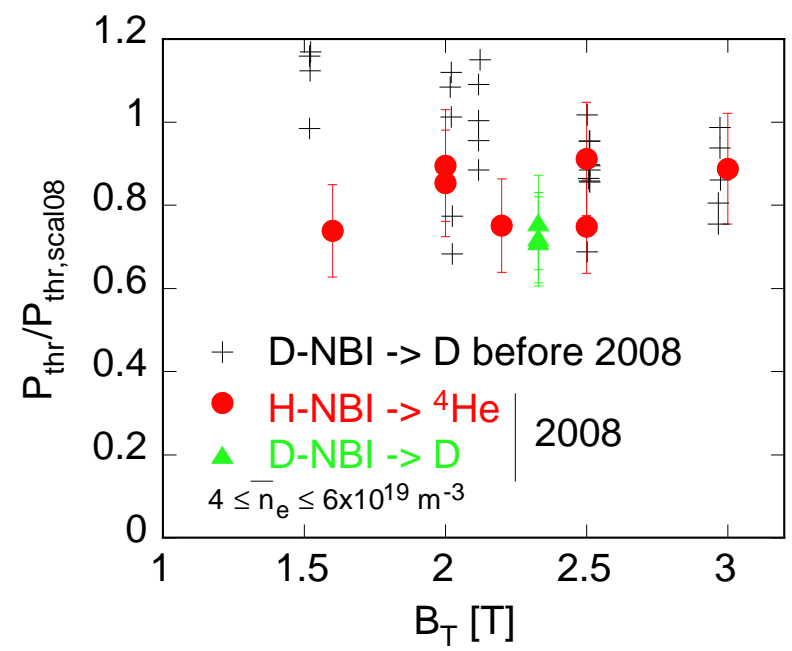

Figure 3. Power threshold normalized to scaling assuming deuterium, versus magnetic field for a narrow density range indicated in the plot. The effect of the density variation is corrected by the scaling.

The density dependence of $P_{t h r}$ has been investigated in 2008 with ECH in both D and ${ }^{4} \mathrm{He}$ discharges with the same plasma parameters $\left(I_{p}=1.0 \mathrm{MA}, B_{T}=2.3\right.$, T $\left.q_{95} \approx 4.5\right)$. For comparison between heating methods, points for $P_{t h r}$ have also been obtained with NBI.

The results for the density dependence, Fig. 4, clearly exhibit a minimum of $P_{t h r}$ at $n_{\min } \approx 4.5 \times 10^{19} \mathrm{~m}^{-3}$. The minimum is very similar for the two gases. The fact that also the values of $P_{t h r}$ are the same over the whole density range is in contrast to our previous results (Sect. 2) and to the JET results. The deuterium points with ECH have mainly been obtained in May 2008. Motivated by the unexpected lack of difference between $P_{t h r}$ in D and ${ }^{4} \mathrm{He}$, in October, we performed further experiments in D with ECH and with D-NBI, which confirmed the results. There is so far no explanation for the discrepancy between the previous and the new results. As possible influence, it should be underlined that the 2008 experiments have been carried out with the full tungsten wall instead of graphite for the previous data. Also, a somewhat different plasma shape has been used which better fits the divertor geometry. The line plotted in Fig. 4 shows that both the deuterium and helium points obtained in these new series are about $20 \%$ below the scaling in its region of validity $\left(\bar{n}_{e} \geq 4 \times 10^{19} \mathrm{~m}^{-3}\right)$. In fact, a comparison with our previous data in deuterium indicates that the 2008 points are just at 


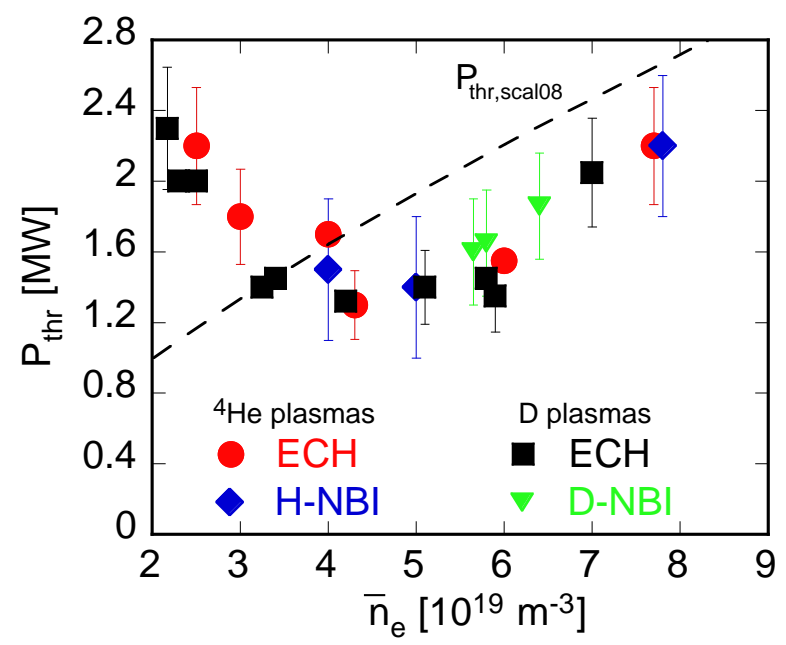

Figure 4. Power threshold versus density for $\mathrm{D}$ and ${ }^{4} \mathrm{He}$, all points from 2008. The dashed line is $P_{t h r, s c a l 08}$ for the corresponding plasma conditions.

the lower boundary of all the previous $P_{t h r}$ values. Finally, it is also worth-noting that no measurable difference can be detected between ECH and NBI.

We collected a complete set of edge data at the LH transition. The first analyses suggest that the edge electron pressure at the LH transition is independent of density and gas [12]. A complete analysis of this aspect will be published elsewhere.

\subsection{New confinement data in ${ }^{4} \mathrm{He}$}

To compare the experimental confinement time in helium, we use the usual ITERH98y2 scaling expression, assuming deuterium. The fast ions corrections required to estimate the experimental thermal confinement time were assumed to be the same as in deuterium, which introduces additional uncertainties up to $10 \%$. Our normalized confinement data, $\tau_{E} / \tau_{\text {ITERH } 98 y 2}$, in helium for H-NBI, D-NBI and ECH are summarized in Fig. 5 versus density. For the three datasets, $\tau_{E} / \tau_{\text {ITERH } 98 y 2}$ decreases with density, with different strengths characterized by a power law indicated in the figure for comparison with $\propto n_{e}^{0.41}$ of ITERH98y2. This property of the density dependence is not specific to helium plasmas and is also generally observed in deuterium plasmas, see e.g. [13], indicating that the actual density dependence is weaker than that of the scaling. The significant difference between D-NBI and H-NBI may be attributed to the influence of helium purity. This is supported by the fact that the ECH points lie in-between and that all datasets converge at high density. It should be pointed out that the uncertainties on the corrections for the fast ions effects mentioned above might also play a role.

During the 2008 campaign we performed D and ${ }^{4} \mathrm{He} H$-mode discharges, heated by D$\mathrm{NBI}$ and $\mathrm{H}-\mathrm{NBI}$ respectively, with the same shape, at $I_{p}=1 \mathrm{MA}, q_{95} \approx 4.5$ and with a heating power of 3.4 MW. The ELM frequency in D was about $50 \mathrm{~Hz}$ whereas it was about $300 \mathrm{~Hz}$ in ${ }^{4} \mathrm{He}$. This led to a higher density in D compared to that in ${ }^{4} \mathrm{He}$. In these discharges, the 


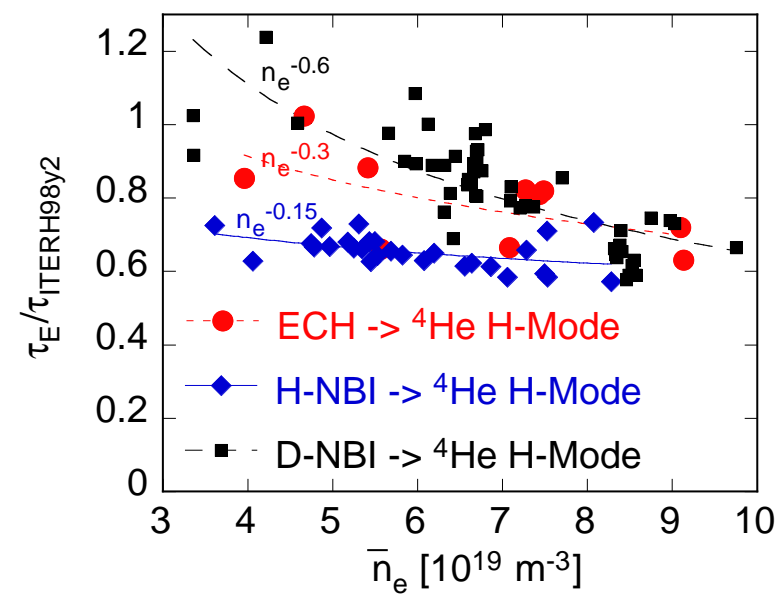

Figure 5. Density dependence of the helium confinement data. The circles and diamonds are from 2008, the squares from previous data. The lines are power fits.

temperature profiles were very similar for both gases with $T_{e} \approx T_{i}$. Taking into account a rough estimate of $Z_{e f f}$ and impurity content, the ion density can be deduced and the total pressure calculated, see Fig. 6 . The pressure in ${ }^{4} \mathrm{He}$ is lower than that in D by about $30 \%$, as also shown in Fig. 6 by the ratio of the pressure in the two gases. This effect is mainly due to $\mathrm{Z}=2$ of the helium ions with an additional contribution to the difference arising from the lower pedestal pressure in helium, likely linked with the ELM behaviour. This is consistent with the different $\tau_{E}$ values in the two gases, $\tau_{E} / \tau_{\text {ITERH98y2 }}$ being $\approx 1$ in $\mathrm{D}$ and $\approx 0.65$ in ${ }^{4} \mathrm{He}$, as also indicated by Fig. 5.

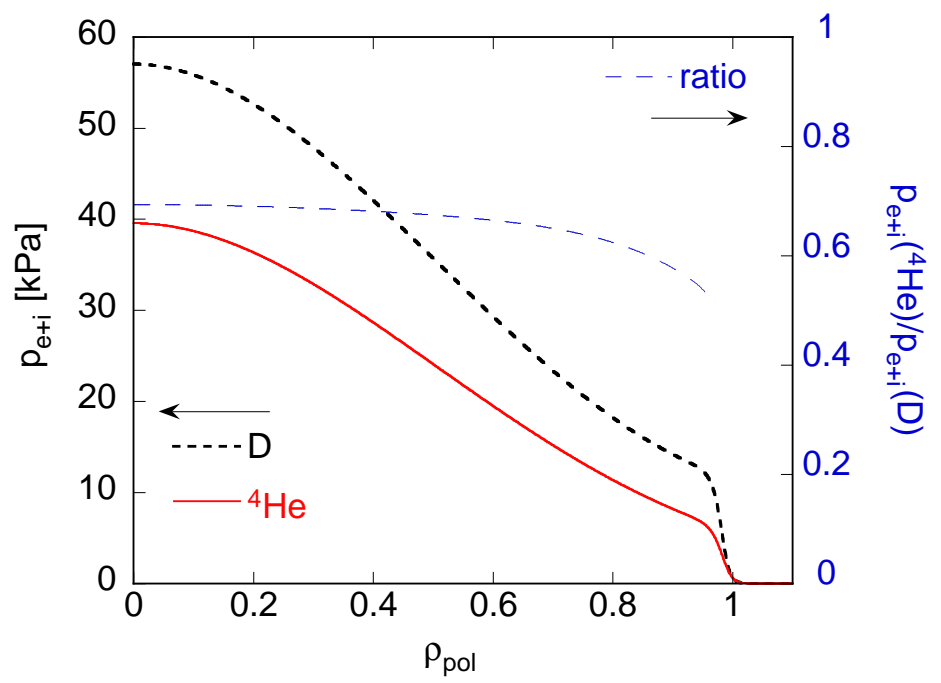

Figure 6. Plasma pressure in $\mathrm{D}$ and ${ }^{4} \mathrm{He}$ : total pressure profiles in the two gases and their ratio.

The different confinement times in ${ }^{4} \mathrm{He}$ and $\mathrm{D}$ have a completely different origin than the well-known isotope effect between $\mathrm{H}$ and $\mathrm{D}$. In the case of hydrogen, the temperature profiles 
are lower than in D due to a higher heat transport as investigated in ASDEX Upgrade, [14].

\section{Conclusion}

As a prompt response to requests for ITPA Joint Experiments, H-mode power threshold and confinement studies have been carried out in ASDEX Upgrade in both helium and deuterium plasmas. The new results indicate that the power threshold in the two gases is very similar, in contrast to previous data from ASDEX Upgrade and JET. The reason for this difference remains to be understood in further experiments in 2009. In any case, the power threshold in helium is clearly lower than in hydrogen which is favorable for ITER. It should be underlined that a metallic inner wall, tungsten in our case, has no deleterious effect on the power threshold.

In agreement with previous data from ASDEX Upgrade and JET, the global confinement time in helium is about $30 \%$ lower than in deuterium, mainly caused by $\mathrm{Z}=2$ in helium. From the point of view of power threshold and confinement, helium appears to be a better choice than hydrogen for the non-nuclear phase of ITER. However, it should be underlined that the expected higher ELM frequency might not allow to test ELM control methods.

It must be underlined that the factor of 2 between electron and ion pressure in helium is an interesting situation to extend $\mathrm{H}$-mode transition and pedestal physics studies. It could help sorting out the respective role of ions and electrons and may widen the understanding of the ELM physics, for instance in comparison with impurity seeding experiments. Therefore, comparison between deuterium and helium plasmas, which is of great interest for future tokamak operation and physics understanding, should be intensified.

\section{References}

[1] MARTIN, Y. R. et al., Journal of Physics: Conference Series 123 (2008) 012033.

[2] ASDEX Team, Nucl. Fusion 29 (1989) 1959.

[3] RYTER, F. and H-Mode Database Working Group, Nucl. Fusion 36 (1996) 1217.

[4] LACKNER, K. et al., Plasma Phys. Controlled Fusion 36 (1994) B79.

[5] RIGHI, E. et al., Nucl. Fusion 39 (1999) 309.

[6] MCDONALD, D. C. et al., Plasma Phys. Controlled Fusion 46 (2004) 519.

[7] FIELDING, S. J. et al., Plasma Physics and Controlled Fusion 40 (1998) 731.

[8] FUKUDA, T. et al., Plasma Physics and Controlled Fusion 42 (2000) A289.

[9] ANDREW, Y. et al., Plasma Phys. Controlled Fusion 48 (2006) 479.

[10] SNIPES, J. A. et al., Plasma Current Dependence of the H-mode Threshold Low Density Limit on Alcator C-Mod, in Europhysics Conference Abstracts (CD-ROM), Proc. of the 35th EPS Plasma Physics Conference, Hersonissos (2008) Paper P1.074.

[11] ITER Physics Basis, Chap. II, page 2208, Table 5, Nucl. Fusion 39 (1999).

[12] RYTER, F. et al., Paper PD/1-1, $22^{\text {nd }}$ Fusion Energy Conference, Geneva (2008).

[13] STOBER, J. et al., Plasma Phys. Controlled Fusion 42 (2000) A211.

[14] STOBER, J. et al., Confinement and transport studies at high power in ASDEX Upgrade, in Europhysics Conference Abstracts (CD-ROM), Proc. of the 1998 ICPP \& 25th EPS Conference on Controlled Fusion and Plasma Physics, Praha, 1998, edited by PAVLO, P., volume 22C, pages 476-479, Petit-Lancy, 1998, EPS. 